\title{
Low-temperature breakdown of antiferromagnetic quantum critical behavior in FeSe
}

\author{
V. Grinenko, ${ }^{1,2,{ }^{*}}$ R. Sarkar, ${ }^{1}$ P. Materne, ${ }^{1}$ S. Kamusella, ${ }^{1}$ A. Yamamshita, ${ }^{3}$ Y. Takano,${ }^{3}$ Y. Sun, ${ }^{4}$ T. Tamegai, ${ }^{4}$ D. V. Efremov, ${ }^{2}$ \\ S.-L. Drechsler, ${ }^{2}$ J.-C. Orain, ${ }^{5}$ T. Goko, ${ }^{5}$ R. Scheuermann, ${ }^{5}$ H. Luetkens, ${ }^{5}$ and H.-H. Klauss ${ }^{1}$ \\ ${ }^{1}$ Institute for Solid State and Materials Physics, TU Dresden, D-01069 Dresden, Germany \\ ${ }^{2}$ IFW Dresden, Helmholtzstrasse 20, D-01069 Dresden, Germany \\ ${ }^{3}$ National Institute for Materials Science (NIMS-MANA), 1-2-1 Sengen, Tsukuba 305-0047, Japan \\ ${ }^{4}$ The University of Tokyo, 7-3-1 Hongo, Bunkyo-ku, Tokyo 113-8656, Japan \\ ${ }^{5}$ Laboratory for Muon Spin Spectroscopy, Paul Scherrer Institute, CH-5232 Villigen PSI, Switzerland
}

(Received 25 December 2017; revised manuscript received 21 February 2018; published 3 May 2018)

\begin{abstract}
A nematic transition preceding a long-range spin density wave antiferromagnetic phase is a common feature of many parent compounds of Fe-based superconductors. However, in the FeSe system with a nematic transition at $T_{\mathrm{s}} \approx 90 \mathrm{~K}$, no evidence for long-range static magnetism is found down to very low temperatures. The lack of magnetism is a challenge for the theoretical description of FeSe. We investigated high-quality single crystals of FeSe using high-field (up to $9.5 \mathrm{~T}$ ) muon spin rotation ( $\mu \mathrm{SR}$ ) measurements. The $\mu$ SR Knight shift and the bulk susceptibility linearly scale at high temperatures but deviate from this behavior around $T^{*} \sim 10-20 \mathrm{~K}$, where the Knight shift exhibits a kink. In the temperature range $T_{\mathrm{s}} \gtrsim T \gtrsim T^{*}$, the muon spin depolarization rate shows a quantum critical behavior $\Lambda \propto T^{-0.4}$. The observed critical scaling indicates that FeSe is in the vicinity of an itinerant antiferromagnetic quantum critical point. Below $T^{*}$ the quantum critical behavior breaks down. We argue that this breakdown is caused by a temperature-induced Lifschitz transition.
\end{abstract}

DOI: 10.1103/PhysRevB.97.201102

Understanding the interplay between nematic, magnetic, and superconducting orders is one of the key problems of Febased superconductors [1]. Many theoretical and experimental efforts have been applied to shed light on the nature of the broad nematic region of the FeSe system [1-5]. In this system, superconductivity with a transition temperature $T_{\mathrm{c}} \approx 9 \mathrm{~K}$ develops from a paramagnetic phase with a nematic transition temperature $\left(T_{\mathrm{s}} \approx 90 \mathrm{~K}\right)$ without any evidence of long-range static magnetism down to low $T$.

The transport properties of FeSe show a complex magnetic field and temperature dependence below $T_{\mathrm{S}}$ [6-10], which cannot only be accounted for by the anisotropic scattering in the nematic phase [11]. The analysis of the transport data indicates essential changes in the electronic structure [6-9] and/or hints to enhanced spin fluctuations (SFs) and the formation of a pseudogap above $T_{\mathrm{c}}[10,12,13]$. Nuclear magnetic resonance (NMR) measurements revealed that FeSe exhibits a quite different SF spectrum as compared with other Fe-based superconductors. The $T$ dependence of the spin-lattice relaxation rate $1 / T_{1} T$ is only enhanced below $T_{\mathrm{s}}[12-15]$. However, recent inelastic neutron scattering measurements revealed that strong Néel antiferromagnetic (AF) SFs at $Q_{\mathrm{N}}=[\pi, \pi]$ above $T_{\mathrm{S}}$ exist at high energies [16]. Moreover, the SFs at $Q_{\mathrm{N}}$ are suppressed and stripe $\mathrm{AF}$ SFs at $Q_{\mathrm{S}}=[\pi, 0]$ are strongly enhanced below $T_{\mathrm{S}}$. In contrast to the Néel SFs at high temperatures, the stripe SFs have a noticeable low-energy tail [17], which is accessible in NMR experiments. In general, experimental and theoretical investigations indicate that $\mathrm{FeSe}$ is on the border of magnetism.

\footnotetext{
*v.grinenko@ifw-dresden.de
}

However, the energy scale which can characterize the distance to a corresponding magnetic quantum critical point $(\mathrm{QCP})$ and the reasons for the pseudogaplike behavior above $T_{\mathrm{c}}$ seen in many experiments in stoichiometric FeSe are unclear.

To get a deeper insight into the magnetic properties of $\mathrm{FeSe}$ in the nematic state, we preformed high-resolution muon spin rotation/relaxation $(\mu \mathrm{SR})$ experiments. The positively charged spin-1/2 muon is one of the most sensitive probes to measure local internal magnetic fields at the muon stopping sites located usually at places of maximal electronic density in metals. However, the exact occupation of possible stopping sites can be hardly predicted, which complicates the analysis of $\mu$ SR data. The unique high-field $\mu$ SR instrument in PSI Villigen allowed us to resolve the individual muon stopping sites in high-quality FeSe single crystals. This provides access to the Knight shift and the muon depolarization rate specific to the muon site. Our local probe measurements revealed that, in accord with previous results, FeSe has no static magnetism in the main sample volume but low-energy SFs lead to non-Fermi-liquid (non-FL) behavior with a cutoff temperature $T^{*} \sim 10-20 \mathrm{~K}$, indicating a close proximity to a magnetic QCP.

High-quality FeSe single crystals were grown by the vapor transport method, which is described in Refs. [8,9]. The crystals used in the $\mu$ SR experiments were characterized by various techniques [18]. For the $\mu \mathrm{SR}$ experiments, crystals with a $T_{\mathrm{c}} \approx 9 \mathrm{~K}$ were selected by magnetization measurements. Hightransversal-field (TF) $\mu \mathrm{SR}$ measurements were performed at the HAL-9500 spectrometer and zero-field (ZF) and low-field experiments (given in the Supplemental Material (SM) [18]) were done at the DOLLY spectrometer (PSI, Villigen) on a mosaic of the selected crystals with a total mass of about $12 \mathrm{mg}$ [19]. For measurements using the HAL-9500 spectrometer the 

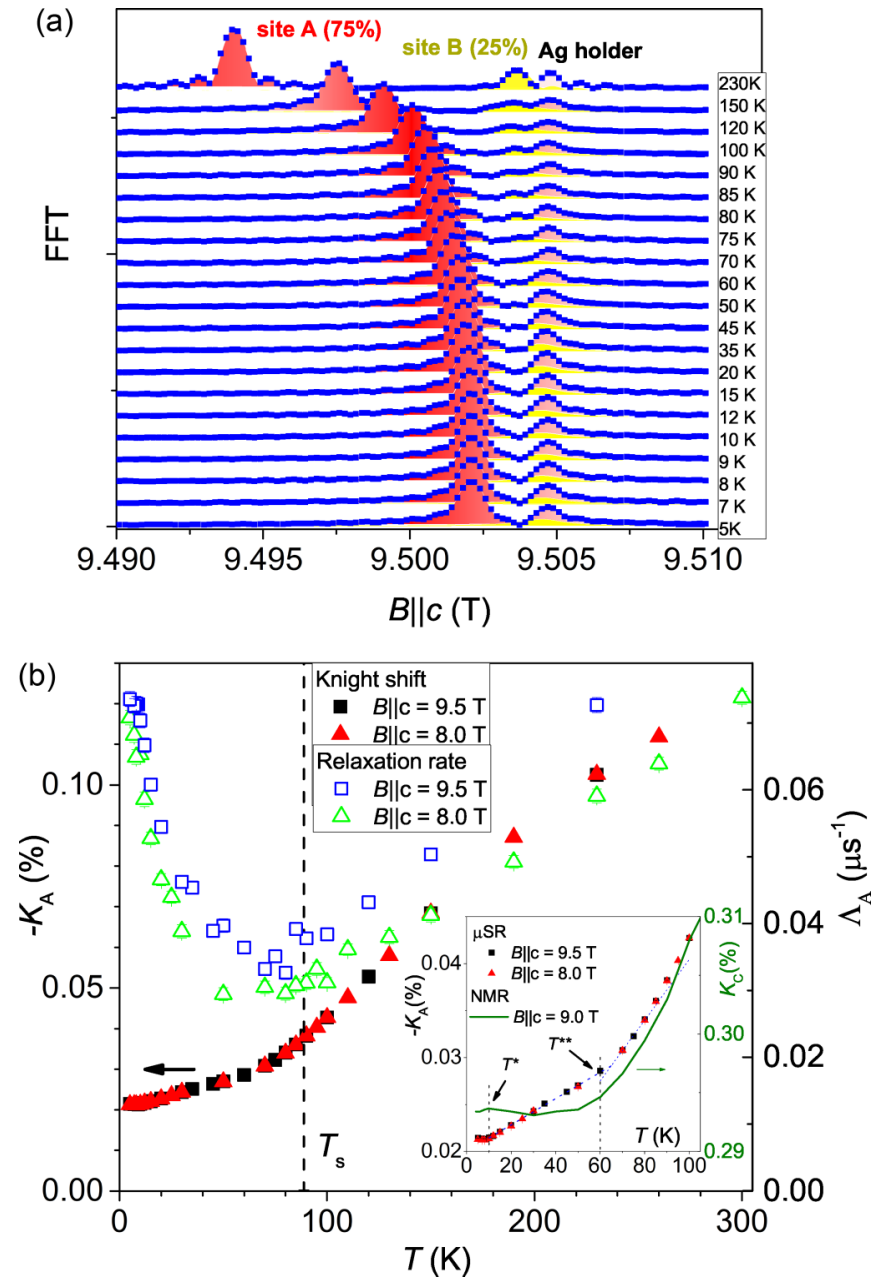

FIG. 1. (a) FFT of the TF $\mu$ SR time spectra measured in $B \| c=$ 9.5 $\mathrm{T}$ at different temperatures for FeSe. The peaks denoted as sites $\mathrm{A}$ and $\mathrm{B}$ are related to muons stopping in the sample. The peak denoted as Ag holder is a signal from muons stopping in the silver sample holder. (b) The $\mu$ SR Knight shift (left axis) and relaxation rate (right axis) of the muons stopping at site A vs $T$ in $B \| c=9.5$ and $8 \mathrm{~T}$. Inset: The same data (left axis) and NMR Knight shift taken from Ref. [14] (right axis) below $100 \mathrm{~K}$.

muon polarization was in the $a b$ plane. The $\mu \mathrm{SR}$ data were analyzed using the MUSRFIT software package [21].

Fast Fourier transformations (FFTs) of the TF- $\mu$ SR spectra measured in a magnetic field $B \| c=9.5 \mathrm{~T}$ and at different $T$ are shown in Fig. 1(a). The FFT spectra consist of three peaks. The right narrow peak corresponds to the Ag sample holder. Its position was used as an internal reference to calculate the absolute value of the Knight shift. The left peak (site A) corresponds to the majority of muons $(\sim 75 \%)$ stopping in the sample. The peak is relatively narrow, just slightly broader than the peak of the $\mathrm{Ag}$, but its position is strongly $T$ dependent. According to Ref. [22], site A is located close to the Fe atomic planes. The position of the broad central peak (site B) is weakly $T$ dependent. By contrast, its width shows a nonmonotonic $T$ dependence. Site B is located within the Se atomic plane and it has a shallow energy minimum as compared to site $\mathrm{A}$ [22]. Therefore, the muon depolarization rate for site B is less affected by the interaction of muons with the Fe spins, which explains the significantly smaller Knight shift. On the other hand, the larger linewidth can be caused by the interaction with diluted randomly distributed Se nuclear spins [23]. A shallow energy minimum results in muon diffusion at high $T$ suppressing depolarization due to static nuclear moments. Below $70 \mathrm{~K}$ the diffusion slows down and the depolarization rate in $\mathrm{ZF}$ data saturates around $\Lambda_{\mathrm{B}} \sim 0.35 \mu \mathrm{s}^{-1}$ [18]. Depolarization due to diluted magnetic impurities [24] can be excluded since in this case the muons stopping at site A should be also affected by stray fields. However, we did not find any noticeable static contribution to the depolarization rate of site A. A very low impurity concentration in our sample is also consistent with the Mössbauer data given in the SM [18]. This analysis imposes an upper limit for the possible magnetic moment of $0.03 \mu_{B}$ and an impurity $\mathrm{Fe}$ concentration of 0.1 at. \%. Based on the $\mu \mathrm{SR}$ and Mössbauer data, we exclude the presence of internal magnetic fields which could be associated with an AFM state in the main sample volume (site A) down to $T_{\mathrm{c}}$. For further analysis we consider the signal from muons stopping at site A as representative of the main sample volume.

To obtain the Knight shift values and the relaxation rates specific to muon sites, we analyzed the high-TF $\mu$ SR spectra by a sum of three contributions,

$$
P(t)=\sum_{i=1}^{3} P_{\mathrm{i}} \cos \left(2 \pi \nu_{\mathrm{i}} t+\frac{\pi \varphi}{180}\right) \exp \left[-\Lambda_{\mathrm{i}} t\right],
$$

where $\mathrm{i}$ corresponds to site $\mathrm{A}$, site $\mathrm{B}$, and the $\mathrm{Ag}$ sample holder, respectively, and $\phi$ is the phase. The ratio $P_{\mathrm{A}} / P_{\mathrm{B}}=3$ between the fractions of muons stopping at sites $\mathrm{A}$ and $\mathrm{B}$ was found to be nearly field and $T$ independent and therefore it was fixed within the analysis. The fraction $P_{\mathrm{Ag}}$ was field dependent due to the field dependence of the beam spot size. $\Lambda_{\mathrm{i}}$ is the muon depolarization rate (peak width). The results of the fit by Eq. (1) are shown by the filling colors in Fig. 1(a). The obtained $T$ dependence of the $\mu$ SR Knight shift $K_{\mathrm{A}}=\left(v_{\mathrm{A}}-v_{\mathrm{Ag}}\right) / v_{\mathrm{Ag}}$ and the muon depolarization rate $\Lambda_{\mathrm{A}}$ for site $\mathrm{A}$ in $B \| c=9.5$ and $8 \mathrm{~T}$ are shown in Fig. 1(b). All data shown here are corrected by demagnetization effects [25]. $K_{\mathrm{A}}$ (left axis) is field independent and at high $T$ shows a $T$ dependence similar to that observed in ${ }^{77}$ Se-NMR measurements, as shown in the inset of Fig. 1(b) $[14,15]$. It is seen that $K_{\mathrm{A}}$ is only slightly affected by the structural transition at $T_{\mathrm{s}}$, which results in a gradual change of the slope of the $T$ dependence. Below $T_{\mathrm{s}}$, two kinklike features are observed in the $T$ dependence of $K_{\mathrm{A}}$ around $T^{* *} \sim 60 \mathrm{~K}$ and $T^{*} \sim 10 \mathrm{~K}$. Note that the feature at $T^{*}$ is not caused by the bulk superconducting transition since $T_{\mathrm{c}}$ is about $5 \mathrm{~K}$ in $B \| c=9$ T, according to the specific heat data [18].

The anomalous behavior of the electronic system at low $T$ is seen in Fig. 2 from the plot of $K_{\mathrm{A}}$ versus the bulk molar susceptibility $\chi_{\mathrm{m}}$ with $T$ as an implicit parameter. The $T$ dependence of $\chi_{\mathrm{m}}$ is given in the SM [18]. In NMR experiments, the Knight shift is usually linear with the susceptibility and one can extract the orbital $T$-independent contribution to the Knight shift from the intercept and the hyperfine coupling from the slope of the linear fit [26]. At high $T$ above $T_{\mathrm{s}}, K_{\mathrm{A}}=A_{0} \chi_{\mathrm{m}}+K_{\text {orb }}$ is linear with $\chi_{\mathrm{m}}$, resulting in a $T$-independent coupling constant $A_{0} \approx-3.3 \mathrm{~T} /$ f.u. $\mu_{\mathrm{B}}$ and a positive $K_{\text {orb }} \approx 0.02 \%$, which can be interpreted as a $T$-independent nuclear and orbital contribution in analogy with NMR. The parameter $A_{0}$ accounts 


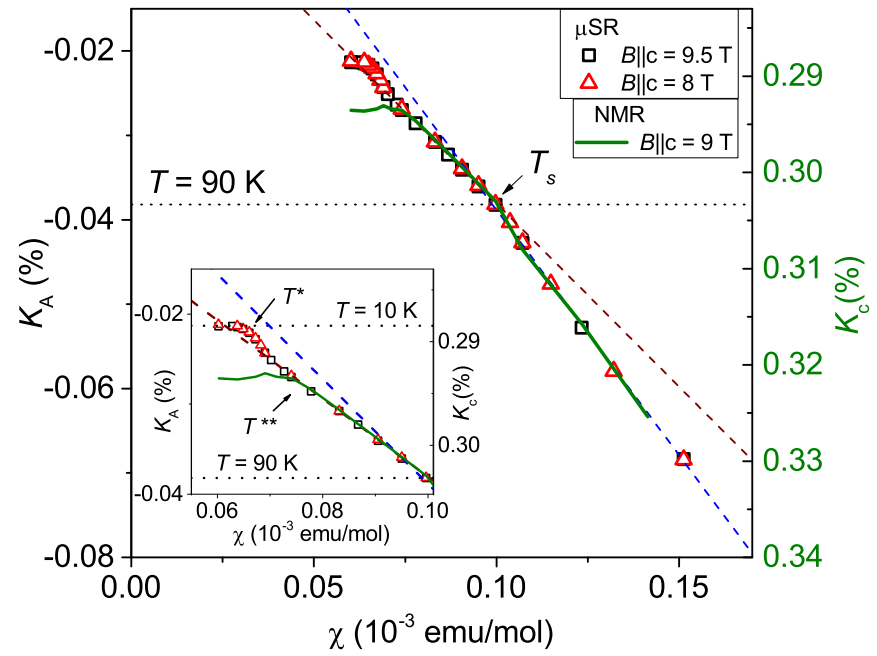

FIG. 2. The $\mu$ SR Knight shift $K_{\mathrm{A}}$ (left axis) and NMR Knight shift $K_{\mathrm{c}}$ taken from Ref. [14] (right axis) vs bulk molar susceptibility $\chi_{\mathrm{m}}$ of FeSe. Lines are linear fits of the data. Inset: The same data below $100 \mathrm{~K}$.

for all $T$-dependent terms. In the case of FeSe, the dipolar, hyperfine, and Fermi contact contributions to the Knight shift can be $T$ dependent even above $T_{\mathrm{s}}$ due to a strong $T$ dependence of the chemical potential [27]. The nematic transition results in the reduction of $A_{0} \approx-2.4 \mathrm{~T} /$ f.u. $\mu_{\mathrm{B}}$ and $K_{\text {orb }} \approx 0.005 \%$. However, $K_{\mathrm{A}}$ remains linear with $\chi_{\mathrm{m}}$. The linear relationship is violated only around $T^{*}$, indicating a significant $T$ variation of $A_{0}$ and $K_{\text {orb }}$. The NMR $K_{\mathrm{c}}$ has the same behavior as the $\mu \mathrm{SR} K_{\mathrm{A}}$ down to $T^{* *}$ and becomes nearly constant at low $T$. The loss of linear proportionality between the NMR $K_{\mathrm{c}}$ and $\chi_{\mathrm{m}}$ below $T^{* *}$ is attributed to a strong reduction of the Se hyperfine coupling constant $A_{\mathrm{Se}}$. In contrast, the higher sensitivity of the $\mu \mathrm{SR} K_{\mathrm{A}}$ to $\chi_{\mathrm{m}}$ is related to the smaller distance of the muon site A to the Fe atomic layers as compared to Se atoms used in NMR experiments [22].

The observation of kinks in the $T$ dependence of $K_{\mathrm{A}}$ [Fig. 1(b)], the broken linear relationship between $K_{\mathrm{A}}$ and $\chi_{\mathrm{m}}$ (Fig. 2), and features in the electrical resistivity [18] and Hall coefficient [10] indicate that $T^{*}$ and $T^{* *}$ correspond to a reduction of the density of states (DOS), as suggested previously in Refs. $[10,12,13]$. The effects were attributed to the formation of the pseudogap due to the charge density wave phase competing with the magnetism or preformed Cooper pairs, respectively. Alternatively, the reduction of the DOS can be caused by a $T$-induced Lifshitz transition. Indeed, a strong $T$ dependence of the Fermi energies of individual bands was found in recent angle-resolved photoemission spectroscopy (ARPES) experiments. At high $T$ above $T_{\mathrm{s}}$, the Fermi surface (FS) consists of two holelike $(h)$ and two electronlike $(e l)$ FS sheets. However, one $h$ FS pocket (around the $Z$ point) sinks below the Fermi level with the reduction of $T$ below $T_{\mathrm{s}}$, according to both ARPES and Shubnikov-de Haas oscillations measurements [28,29]. The situation concerning the el FS sheets at low $T$ is controversial. The Shubnikov-de Haas oscillations revealed one $e l$ thin cylinder only. However, some ARPES data indicate a complex structure around the $M$ point of the Brillouin zone with two el FS pockets. Below $T_{\mathrm{s}}$ the

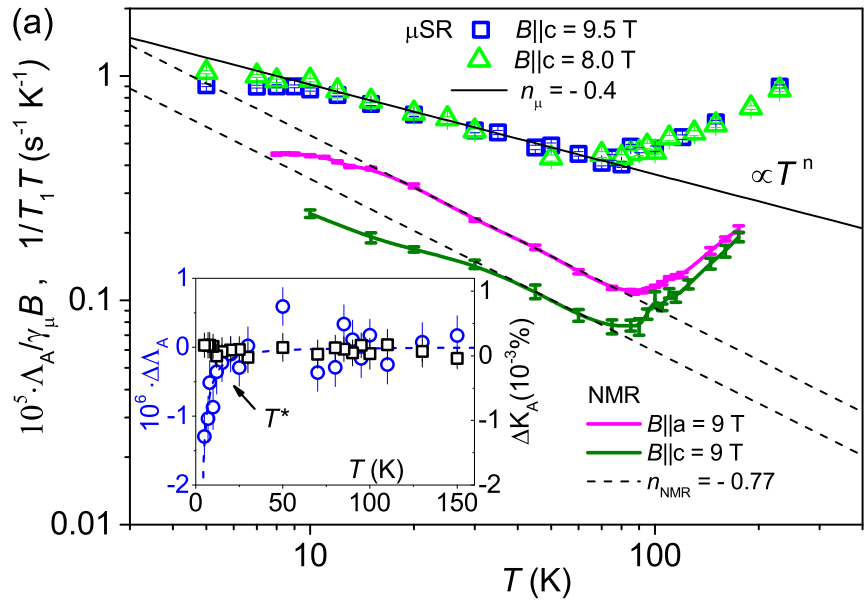

(b)

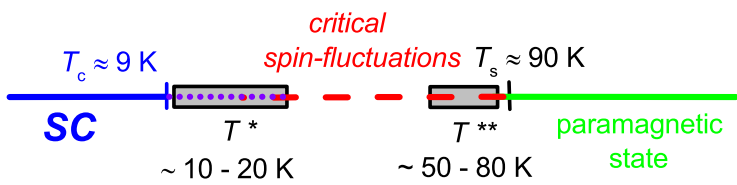

FIG. 3. (a) Log-log plot of the muon depolarization rate $\Lambda_{\mathrm{A}} / \gamma_{\mu} B$ and the NMR $1 / T_{1} T$ taken from Ref. [15] vs $T$ for FeSe. Inset: The muon depolarization rate $\Delta \Lambda_{\mathrm{A}}=\Lambda_{\mathrm{A}}\left(B_{2}\right) / \gamma_{\mu} B_{2}-\Lambda_{\mathrm{A}}\left(B_{1}\right) / \gamma_{\mu} B_{1}$ (left axis), where $B_{1}=8 \mathrm{~T}$ and $B_{2}=9.5 \mathrm{~T}$ and the Knight shift $\Delta K_{\mathrm{A}}=K_{\mathrm{A}}(9.5 \mathrm{~T})-K_{\mathrm{A}}(8 \mathrm{~T})$ (right axis) vs $T$. (b) A schematic phase diagram of FeSe. The gray boxes show the temperature regions attributed to putative Lifshitz transitions.

energy distribution curves (EDCs) result in four peaks for the twinned crystals $[27,30]$. In particular, one of the peaks in the EDC touches the Fermi level within experimental resolution at low $T$ [27]. In conclusion, the experimental data indicate the possibility of two distinct $T$-induced Lifshitz transitions below $T_{\mathrm{s}}$.

To estimate an impact of these Lifshitz transitions on the DOS we analyzed the calculated band structure obtained using the full potential local orbital band structure package (FPLO) [31]. In our calculations we observed that the experimental asymmetric shift of the Fermi energy of the $h$ and $e l$ bands with $T$ results in noticeable anomalies in the DOS when the Fermi energy touches the bottom or top of the bands [18]. The change in the DOS affects both spin and orbital contributions to the Knight shift and therefore may be responsible for the observed kinks in $K_{\mathrm{A}}$ at $T^{*}$ and $T^{* *}$, and for the nonlinear $K_{\mathrm{A}}$ vs $\chi_{\mathrm{m}}$ plot around $T^{*}$ (Fig. 2).

To get deeper insight into the effects around $T^{*}$ we analyzed the $T$ dependence of the muon depolarization rate $\Lambda_{\mathrm{A}}$ [Fig. 1(b), right axis] obtained using Eq. (1). The log-log plot of the $T$ dependence of the dimensionless $\Lambda_{\mathrm{A}} / \gamma_{\mu} B$ with $\gamma_{\mu}=2 \pi \times 135.53 \mathrm{MHz} / \mathrm{T}$ and that of the NMR $1 / T_{1} T$ [15] are shown in Fig. 3(a). Both quantities decrease at high $T$ and increase below $T_{\mathrm{s}}$. This behavior was interpreted as a competition between two types of SFs [32]. Both $\Lambda_{\mathrm{A}}$ and $1 / T_{1} T$ for $B \| a$ follow a critical power-law behavior below $T_{\mathrm{s}}$ with the exponents $n_{\mu} \approx-0.4$ and $n_{\mathrm{NMR}} \approx-0.77$, correspondingly. The $n_{\mu} \approx-0.4$ is close to the theoretical value $-v(z-1) \approx-0.5$ [33] expected for a quantum critical behavior of itinerant AF with the correlation-length exponent 
$v=1 / 2$ and the dynamical exponent $z=2$ [34]. The exponent $n_{\mathrm{NMR}} \approx-0.77$ in $1 / T_{1} T$ is close to the theoretical value $-3 / 4$ for the three-dimensional (3D) spin density wave (SDW) phase found in heavy fermion systems [35]. The power-law scaling of $\Lambda_{\mathrm{A}}$ indicates that the linewidth of site $\mathrm{A}$ in our TF data [Fig. 1(a)] is dominated by SFs [23]. In this case, $\Lambda_{\mathrm{A}}(T) \propto$ $T \int A(q) \chi(q) F(q, \omega) d^{D} q$, where $A(q)$ is the $q$-dependent hyperfine coupling constant, $D$ is the dimensionality, $\chi(q)$ is the $q$-dependent susceptibility, $F(q, \omega)$ is the spectral-weight function, and $\omega$ is the frequency, in our case, $\omega=\gamma_{\mu} B$ [33]. Thus, it is expected that $\Lambda_{\mathrm{A}} / T \propto 1 / T_{1} T$. The observed different exponents of $\Lambda_{\mathrm{A}} / T$ and $1 / T_{1} T$ for $B \| a$, and the deviation from the power-law behavior of $1 / T_{1} T$ for $B \| c$ can be attributed to the $T$-dependent $A_{\mathrm{Se}}(q)$ [36].

The non-FL behavior below $T_{\mathrm{s}}$ is also evidenced by the quasilinear dependence of the electrical resistivity $\rho=\rho_{0}+$ $A T+B T^{2}$ with $A \gg B T$ [18]. A linear $T$ dependence is usually expected in the quantum critical region, which has a $\mathrm{V}$-shaped form in the phase diagram separated by the crossover temperature from the FL state [37]. In this sense, $T^{*}$ can be interpreted as the crossover temperature. One might relate the anticipated QCP to the stripe AF order observed in FeSe under hydrostatic pressure [38-40]. Indeed, the resistivity is perfectly linear below $T_{\mathrm{s}}$ at a small applied pressure $P_{\mathrm{c}} \sim 0.2-0.5 \mathrm{GPa}$ [41]. Therefore, our detection of critical SFs in FeSe at zero pressure suggests that the linear resistivity is related to a QCP at the pressure $P_{\mathrm{c}}$. However, in microscopic experiments such as $\mu \mathrm{SR}$ and NMR, a magnetic phase under pressure is observed only at $P \gtrsim 0.8 \mathrm{GPa}$ with a relatively high transition temperature $T_{\mathrm{N}}[38,42,43]$. This behavior suggests that the QCP is avoided. To settle this puzzle, we propose that the QCP at zero $T$ is avoided by the same mechanism, which is responsible for the anomaly at $T^{*}$.

The difference $\Delta \Lambda_{\mathrm{A}}=\Lambda_{\mathrm{A}}\left(B_{2}\right) / \gamma_{\mu} B_{2}-\Lambda_{\mathrm{A}}\left(B_{1}\right) / \gamma_{\mu} B_{1}$ is shown in the inset of Fig. 3(a), where $B_{1}=8 \mathrm{~T}$ and $B_{2}=$ 9.5 T. $\Delta \Lambda_{\mathrm{A}}$ is almost zero above $T^{*}$, indicating that $\Lambda_{\mathrm{A}} \propto$ $B$. This suggests that $F(q, \omega) \propto \omega$ at the measured frequency range, which should be related to the SF spectra of FeSe. $\Delta \Lambda_{\mathrm{A}}$ changes the behavior across $T^{*}$, where the linear relationship between $K_{\mathrm{A}}$ and $\chi_{\mathrm{m}}$ is broken. The negative value of $\Delta \Lambda_{\mathrm{A}}$ indicates a relative narrowing of the lines with the magnetic field. This nonlinear behavior might be caused by the changes in the shape of $F(q, \omega)$. Alternatively, it was proposed that the field dependence originates from the suppression of the $T^{*}$ anomaly by the magnetic field $[12,13]$. The field dependence of $T^{*}$ is expected for both the preformed Cooper pairs and Lifshitz transition scenario (due to Zeeman effects). The formation of Cooper pairs opens a gap at the Fermi level and therefore affects both $1 / T_{1} T$ and the Knight shift. However, $K_{\mathrm{A}}$ around $T^{*}$ is field independent within the error bars [inset of Fig. 3(a)]. Moreover, the anomaly in the electrical resistivity is shifted to higher $T$ with the magnetic field [18]. In contrast, within the Lifshitz transition scenario, the magnetic field should smear the transition that may affect differently the SF spectra and the DOS. A schematic phase diagram is shown in Fig. 3(b).

In conclusion, the unique high-field $\mu \mathrm{SR}$ technique allowed us to investigate the intrinsic static spin susceptibility and spin dynamics of stoichiometric FeSe. Our microscopic investigation indicates that $\mathrm{FeSe}$ is close to the QCP of the SDW phase, presumably located at a small pressure $P_{\mathrm{c}} \sim$ $0.2-0.5 \mathrm{GPa}$. However, the magnetic quantum critical fluctuations are suppressed and the putative QCP at $P_{\mathrm{c}}$ is avoided by a $T$-induced Lifshitz transition of the $e l$ FS of the $x z / y z$ derived bands.

This work was partially performed at Swiss Muon Source $(\mathrm{S} \mu \mathrm{S})$, PSI, Villigen. We acknowledge stimulating interest and fruitful discussions with A. Amato, A. Böhmer, S. Borisenko, M. Kiselev, M. Khodas, S. Kasahara, R. Khasanov, A. Maeda, S. Molatta, U. Rössler, and Q. Si. D.E. thanks U. Nitzsche for technical assistance. This work was supported by the DFG through Grant No. GR 4667 and within the research training group GRK 1621. R.S. and H.H.K. are thankful to DFG for financial assistance through the SFB 1143 for the project C02. S.-L.D. and D.E. thank the VW-Stiftung for partial support.
[1] R. M. Fernandes, A. V. Chubukovand, and J. Schmalian, Nat. Phys. 10, 97 (2014).

[2] J. K. Glasbrenner, I. I. Mazin, H. O. Jeschke, P. J. Hirschfeld, R. M. Fernandes, and R. Valenti, Nat. Phys. 11, 953 (2015).

[3] F. Wang, S. A. Kivelson, and D.-H. Lee, Nat. Phys. 11, 959 (2015).

[4] Y. Yamakawa, S. Onari, and H. Kontani, Phys. Rev. X 6, 021032 (2016).

[5] R.-Q. Xing, L. Classen, M. Khodas, and A. V. Chubukov, Phys. Rev. B 95, 085108 (2017).

[6] K. K. Huynh, Y. Tanabe, T. Urata, H. Oguro, S. Heguri, K. Watanabe, and K. Tanigaki, Phys. Rev. B 90, 144516 (2014).

[7] M. D. Watson, T. Yamashita, S. Kasahara, W. Knafo, M. Nardone, J. Beard, F. Hardy, A. McCollam, A. Narayanan, S. F. Blake, T. Wolf, A. A. Haghighirad, C. Meingast, A. J. Schofield, H. von Lohneysen, Y. Matsuda, A. I. Coldea, and T. Shibauchi, Phys. Rev. Lett. 115, 027006 (2015).
[8] Y. Sun, S. Pyon, and T. Tamegai, Phys. Rev. B 93, 104502 (2016).

[9] Y. Sun, T. Yamada, S. Pyon, and T. Tamegai, Phys. Rev. B 94, 134505 (2016).

[10] S. Rössler, C. Koz, L. Jiao, U. K. Rössler, F. Steglich, U. Schwarz, and S. Wirth, Phys. Rev. B 92, 060505(R) (2015).

[11] M. A. Tanatar, A. E. Böhmer, E. I. Timmons, M. Schütt, G. Drachuck, V. Taufour, K. Kothapalli, A. Kreyssig, S. L. Bud'ko, P. C. Canfield, R. M. Fernandes, and R. Prozorov, Phys. Rev. Lett. 117, 127001 (2016).

[12] S. Kasahara, T. Yamashita, A. Shi, R. Kobayashi, Y. Shimoyama, T. Watashige, K. Ishida, T. Terashima, T. Wolf, F. Hardy, C. Meingast, H. v. Löhneysen, A. Levchenko, T. Shibauchi, and Y. Matsuda, Nat. Commun. 7, 12843 (2016).

[13] A. Shi, T. Arai, S. Kitagawa, T. Yamanaka, K. Ishida, A. E. Böhmer, C. Meingast, T. Wolf, M. Hirata, and T. Sasaki, J. Phys. Soc. Jpn. 87, 013704 (2018). 
[14] S.-H. Baek, D. V. Efremov, J. M. Ok, J. S. Kim, J. van den Brink, and B. Büchner, Nat. Mater. 14, 210 (2015).

[15] A. E. Böhmer, T. Arai, F. Hardy, T. Hattori, T. Iye, T. Wolf, H. v. Löhneysen, K. Ishida, and C. Meingast, Phys. Rev. Lett 114, 027001 (2015).

[16] Q. Wang, Y. Shen, B. Pan, X. Zhang, K. Ikeuchi, K. Iida, A. D. Christianson, H. C. Walker, D. T. Adroja, M. Abdel-Hafiez, Xiaojia Chen, D. A. Chareev, A. N. Vasiliev, and J. Zhao, Nat. Commun. 7, 12182 (2016).

[17] Q. Wang, Y. Shen, B. Pan, Y. Hao, M. Ma, F. Zhou, P. Steffens, K. Schmalzl, T. R. Forrest, M. Abdel-Hafiez, X. Chen, D. A. Chareev, A. N. Vasiliev, P. Bourges, Y. Sidis, H. Cao, and J. Zhao, Nat. Mater. 15, 159 (2016).

[18] See Supplemental Material at http://link.aps.org/supplemental/ 10.1103/PhysRevB.97.201102 for the following: the magnetization (Figs. S1c and S6), specific heat (Figs. S1(d) and S1(e)), electrical resistivity (Fig. S9), and Mössbauer data (Figs. S2 and S3); the low-TF (Figs. S1(a), S1(b), and S1(c)), ZF- and LF(Figs. S4 and S5) $\mu$ SR data; the magnetic susceptibility data (Fig. S6) and the analysis of the magnetic impurity contribution to the susceptibility (Figs. S7 and S8); and the calculated electronic band structure (Figs. S10 and S11).

[19] Due to the small sample thickness only the experiments with the muon beam directed along the sample $c$ axis were performed. To increase the amount of muons stopping in the sample, we used a thin Al degrader, as described in Ref. [20].

[20] V. Grinenko, P. Materne, R. Sarkar, H. Luetkens, K. Kihou, C. H. Lee, S. Akhmadaliev, D. V. Efremov, S.-L. Drechsler, and H.-H. Klauss, Phys. Rev. B 95, 214511 (2017).

[21] A. Suter and B. M. Wojek, Phys. Proc. 30, 69 (2012).

[22] G. Lamura, T. Shiroka, P. Bonfà, S. Sanna, F. Bernardini, R. De Renzi, R. Viennois, E. Giannini, A. Piriou, and N. Emery, J. Phys.: Condens. Matter 25, 156004 (2013).

[23] The static nuclear linewidth is given by $2.2 \% \mathrm{Fe}$ and $7.6 \% \mathrm{Se}$ sites carrying nuclear moments $\mu / \mu_{\mathrm{N}}=0.09024$ and 0.5325 , correspondingly.

[24] F. K. K. Kirschner, F. Lang, C. V. Topping, P. J. Baker, F. L. Pratt, S. E. Wright, D. N. Woodruff, S. J. Clarke, and S. J. Blundell, Phys. Rev. B 94, 134509 (2016).

[25] The $K_{\mathrm{dem}}=4 \pi(1 / 3-N) \rho_{\mathrm{m}} \chi_{\mathrm{m}}$ is the correction for the demagnetization and Lorentz fields (where $N \approx 1$ is the demagnetization factor and $\rho_{\mathrm{m}}=0.0426 \mathrm{~mol} / \mathrm{cm}^{3}$ is the molar density).

[26] D. M. Nisson and N. J. Curro, New J. Phys. 18, 073041 (2016).

[27] Y. Kushnirenko, A. A. Kordyuk, A. Fedorov, E. Haubold, T. Wolf, B. Büchner, and S. V. Borisenko, Phys. Rev. B 96, 100504 (2017).
[28] M. D. Watson, T. K. Kim, A. A. Haghighirad, N. R. Davies, A. McCollam, A. Narayanan, S. F. Blake, Y. L. Chen, S. Ghannadzadeh, A. J. Schofield, M. Hoesch, C. Meingast, T. Wolf, and A. I. Coldea, Phys. Rev. B 91, 155106 (2015).

[29] T. Terashima, N. Kikugawa, A. Kiswandhi, E.-S. Choi, J. S. Brooks, S. Kasahara, T. Watashige, H. Ikeda, T. Shibauchi, Y. Matsuda, T. Wolf, A. E. Böhmer, F. Hardy, C. Meingast, H. v. Löhneysen, M.-T. Suzuki, R. Arita, and S. Uji, Phys. Rev. B 90, 144517 (2014).

[30] A. Fedorov, A. Yaresko, T. K. Kim, Y. Kushnirenko, E. Haubold, T. Wolf, M. Hoesch, A. Grüneis, B. Büchner, and S. V. Borisenko, Sci. Rep. 6, 36834 (2016).

[31] K. Koepernik and H. Eschrig, Phys. Rev. B 59, 1743 (1999); http://www.fplo.de.

[32] A. Kreisel, S. Mukherjee, P. J. Hirschfeld, and B. M. Andersen, Phys. Rev. B 92, 224515 (2015).

[33] A. Yaouanc and P. Dalmas de Reotier, Muon Spin Rotation, Relaxation and Resonance (Oxford University Press, Oxford, UK, 2011).

[34] H. v. Löhneysen, A. Rosch, M. Vojta, and P. Wölfle, Rev. Mod. Phys. 79, 1015 (2007).

[35] S. Kambe, H. Sakai, Y. Tokunaga, T. D. Matsuda, Y. Haga, H. Chudo, and R. E. Walstedt, Phys. Rev. Lett. 102, 037208 (2009).

[36] A strong $T$ variation of the NMR $A(q=0)$ below $T_{\mathrm{s}}$ is suggested from the $K_{\mathrm{c}}$ vs $\chi_{\mathrm{m}}$ plot (inset of Fig. 2).

[37] T. Shibauchi, A. Carrington, and Y. Matsuda, Annu. Rev. Condens. Matter Phys. 5, 113 (2014).

[38] P. S. Wang, S. S. Sun, Y. Cui, W. H. Song, T. R. Li, R. Yu, H. Lei, and W. Yu, Phys. Rev. Lett. 117, 237001 (2016).

[39] B. W. Lebert, V. Balédent, P. Toulemonde, J. M. Ablett, S. Klotz, T. Hansen, P. Rodiere, M. Raba, and J.-P. Rueff, arXiv:1708.04805.

[40] R. Khasanov, Z. Guguchia, A. Amato, E. Morenzoni, X. Dong, F. Zhou, and Z. Zhao, Phys. Rev. B 95, 180504(R) (2017).

[41] J. P. Sun, K. Matsuura, G. Z. Ye, Y. Mizukami, M. Shimozawa, K. Matsubayashi, M. Yamashita, T. Watashige, S. Kasahara, Y. Matsuda, J.-Q. Yan, B. C. Sales, Y. Uwatoko, J.-G. Cheng, and T. Shibauchi, Nat. Commun. 7, 12146 (2016).

[42] M. Bendele, A. Amato, K. Conder, M. Elender, H. Keller, H.-H. Klauss, H. Luetkens, E. Pomjakushina, A. Raselli, and R. Khasanov, Phys. Rev. Lett. 104, 087003 (2010).

[43] P. Wiecki, M. Nandi, A. E. Böhmer, S. L. Bud'ko, P. C. Canfield, and Y. Furukawa, Phys. Rev. B 96, 180502(R) (2017). 